\title{
Treatment with eldecalcitol (ED-71) and raloxifene combined increases cancellous and cortical bone strength in ovariectomized rats.
}

\author{
Sadaoki Sakai, Satoshi Takeda, Ayako Shiraishi, Nobuo Koike, Masahiko Mihara and Koichi Endo \\ Product Research Dept., Chugai Pharmaceutical Co., Ltd., Gotemba, Japan
}

\section{Backgrounds}

-Eldecalcitol (ED-71; ELD), a $2 \beta$-hydroxypropyloxy derivative of $1 \alpha, 25(\mathrm{OH})_{2} \mathrm{D}_{3}$, was approved for treatment of osteoporosis in Japan in 2011.

-ELD significantly reduced the incidence of vertebral and wrist fractures compared with alfacalcidol, a prodrug of $1 \alpha, 25(\mathrm{OH})_{2} \mathrm{D}_{3}$, in a 3-year clinical study [1].

-ELD inhibited osteoclastic bone resorption and increased bone mass more potently than alfacalcidol in ovariectomized (OVX) rats [2].

-Raloxifene (RAL), a selective estrogen receptor modulator, is globally approved for the treatment and prevention of postmenopausal osteoporosis. -There are no reports describing the efficacy of combination treatment of ELD with RAL in osteoporosis patients or in animal models.

[1] Matsumoto T tet al. Bone 49: 605 (2011) [1] Matsumoto T et al. Bone 49: 605 (2011)
[2] Uchiyama $Y$ et al. Bone 30: 582 (2002)

\section{Objective}

To compare the effects of combining ELD and RAL against each monotherapy in osteoporotic rats.

\section{Summary}

The combination treatment with ELD and RAL

$>$ improved bone mechanical strength by suppressing bone turnover and increasing BMD more than either monotherapy

$>$ reduced the rise of blood $\mathrm{Ca}$ and urinary $\mathrm{Ca}$ excretion seen in ELD monotherapy.

$>$ may avoid excessive reduction of bone turnover.

$>$ showed additive effect on inhibition of mouse bone marrow osteoclastogenesis.
Fig. 1 Bone resorption marker

ELD + RAL significantly decreased urinary DPD compared with ELD (4 wks) or RAL (4, 8 and 12 wks) monotherapy.

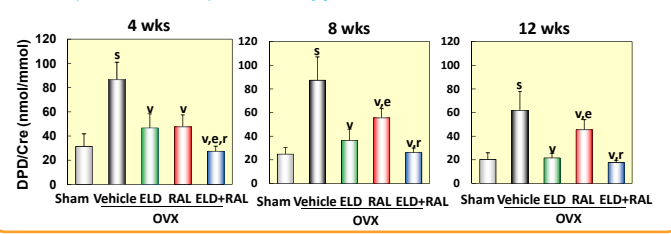

Fig. 2 Bone mineral density

ELD + RAL significantly increased BMD of lumbar spine and femur compared with either monotherapy.
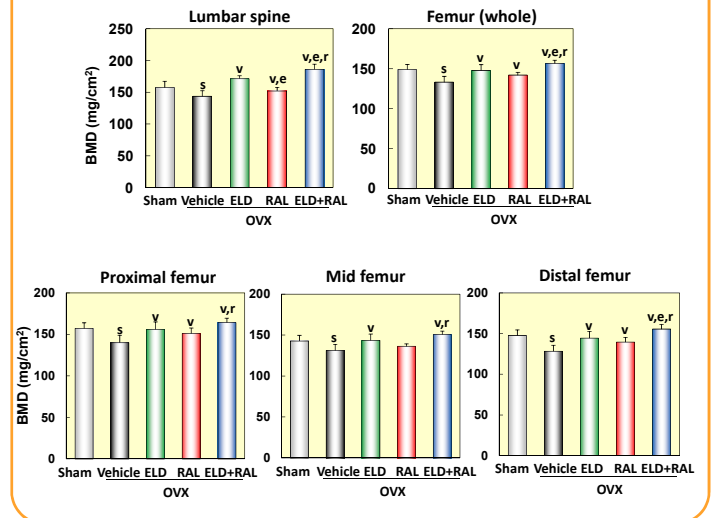

\section{Fig. 5 In vitro osteoclastogenesis}

The combination treatment with ELD and RAL showed additive effect on inhibition of mouse bone marrow osteoclastogenesis.
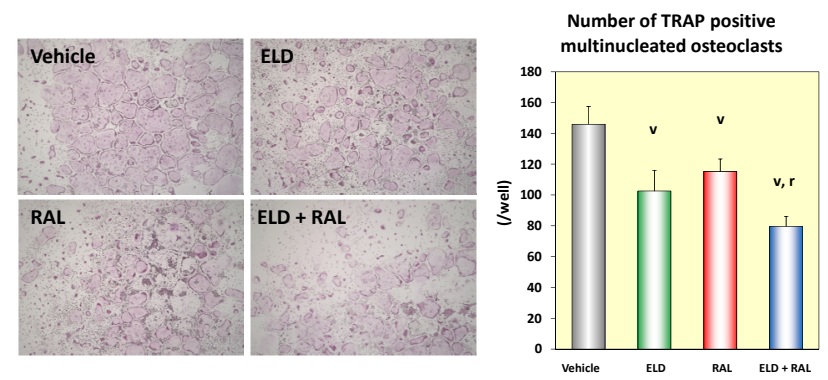

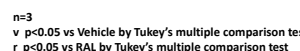

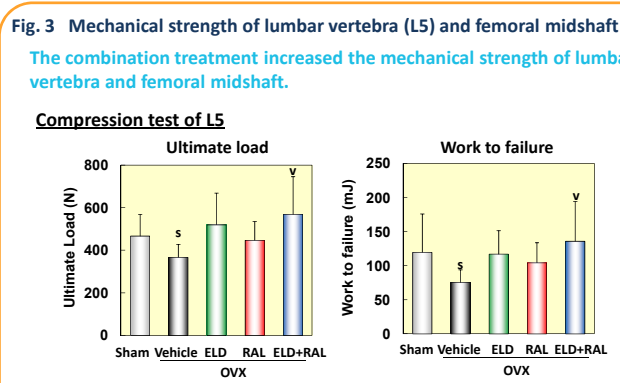

3-point bending test of femoral midshaft

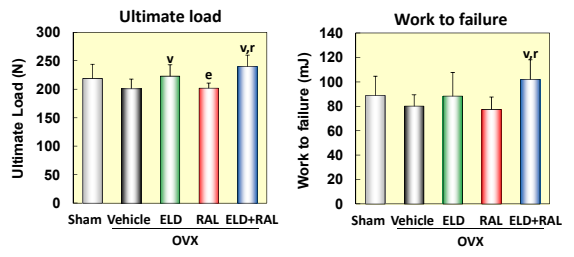

Table 1 Serum $\mathrm{Ca}$ and urinary $\mathrm{Ca} / \mathrm{Cre}(\mathbf{1 2} \mathbf{w k})$

$\mathrm{ELD}+\mathrm{RAL}$ reduced the increase in serum Ca and urinary $\mathrm{Ca} / \mathrm{Cre}$ by ELD.

$$
\text { Sham } \begin{gathered}
\multicolumn{4}{c}{\text { OVX }} \\
\cline { 2 - 5 } \\
\cline { 2 - 4 }
\end{gathered}
$$

Serum Ca (mg/dL) $10.34 \pm 0.31 \quad 9.92 \pm 0.23^{\mathrm{s}} 10.72 \pm 0.36^{\mathrm{V}} \quad 9.62 \pm 0.24^{\mathrm{e}} 10.25 \pm 0.34^{\mathrm{e}, \mathrm{r}}$

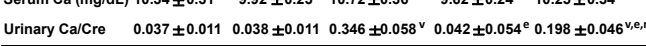

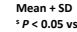

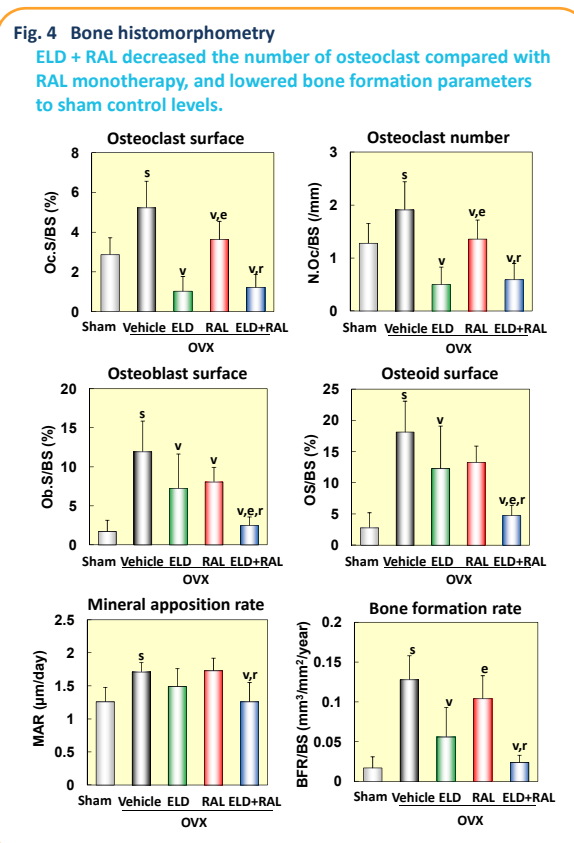

\section{Methods}

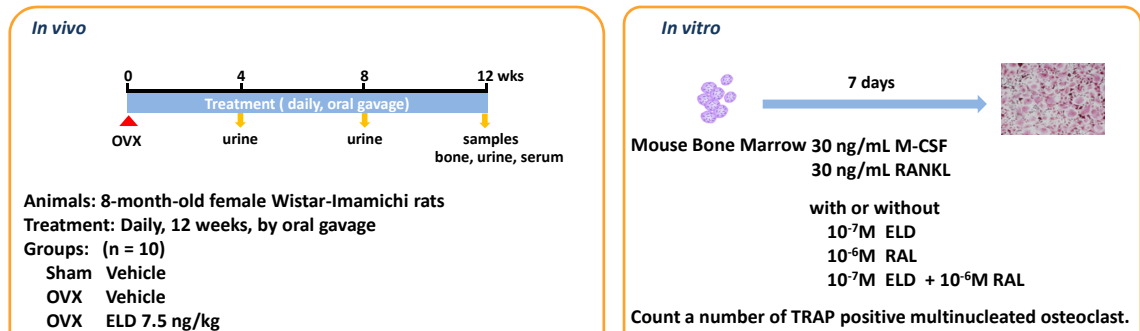

OVX $\quad$ ELD $7.5 \mathrm{ng} / \mathrm{kg}$

OVX $\quad \mathrm{RAL} 0.3 \mathrm{mg} / \mathrm{kg}$

OVX ELD $7.5 \mathrm{ng} / \mathrm{kg}+\mathrm{RAL} 0.3 \mathrm{mg} / \mathrm{kg}$

Measurements:

Bone resorption marker: Urinary deoxypyridinoline (DPD)

BMD: Lumbar spine (L2-L4), femur

Bone biomechanical strength: Lumbar vertebral body (L5), femur

Bone histomorphometry: Lumbar vertebral body (L3)

Serum calcium $(\mathrm{Ca})$, urinary $\mathrm{Ca}$, urinary creatinine (Cre) 